\title{
Ingestion of Lactobacillus strain reduces anxiety and improves cognitive function in the hyperammonemia rat
}

\author{
LUO Jia ${ }^{1,2}$, WANG Tao ${ }^{1}$, LIANG Shan ${ }^{1,2}$, HU Xu $^{1}$, LI Wei ${ }^{1,2} \&$ JIN Feng $^{1 *}$ \\ ${ }^{1}$ Key Laboratory of Mental Health, Institute of Psychology, Chinese Academy of Sciences, Beijing 100101, China; \\ ${ }^{2}$ University of Chinese Academy of Sciences, Beijing 100049, China
}

Received July 26, 2013; accepted September 30, 2013; published online February 18, 2014

\begin{abstract}
Evidence suggests that the hyperammonemia (HA)-induced neuroinflammation and alterations in the serotonin (5-HT) system may contribute to cognitive decline and anxiety disorder during hepatic encephalopathy (HE). Probiotics that maintain immune system homeostasis and regulate the 5-HT system may be potential treatment for HA-mediated neurological disorders in HE. In this study, we tested the efficacy of probiotic Lactobacillus helveticus strain NS8 in preventing cognitive decline and anxiety-like behavior in HA rats. Chronic HA was induced by intraperitoneal injection of ammonium acetate for four weeks in male

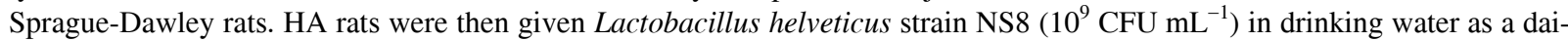
ly supplementation. The Morris water maze task assessed cognitive function, and the elevated plus maze test evaluated anxiety-like behavior. Neuroinflammation was assessed by measuring the inflammatory markers: inducible nitric oxide synthase, prostaglandin E2, and interleukin-1 $\beta$ in the brain. 5-HT system activity was evaluated by measuring 5-HT and its metabolite, 5-HIAA, and the 5-HT precursor, tryptophan. Probiotic treatment of HA rats significantly reduced the level of inflammatory markers, decreased 5-HT metabolism, restored cognitive function and improved anxiety-like behavior. These results indicate that probiotic L. helveticus strain NS8 is beneficial for the treatment of cognitive decline and anxiety-like behavior in HA rats.
\end{abstract}

hyperammonemia, probiotics, cognition, anxiety, neuroinflammation, serotonin

Citation: Luo J, Wang T, Jiang S, Hu X, Li W, Jin F. Ingestion of Lactobacillus strain reduces anxiety and improves cognitive function in the hyperammonemia rat. Sci China Life Sci, 2014, 57: 327-335, doi: 10.1007/s11427-014-4615-4

Patients with liver cirrhosis very frequently develop hepatic encephalopathy (HE), a neuropsychiatric syndrome primarily including cognitive impairment and emotional disorder $[1,2]$. Hyperammonemia (HA) is suggested to be a main factor responsible for these neurological disorders occurring in HE [3-5], and its mechanisms of action are currently under investigation in animal and clinical studies. Data from these studies suggest that HA induced cognitive impairment may be mediated by neuroinflammation [6,7]. Furthermore, these studies support the idea that anxiety disorder, one of the emotional disorders commonly observed in patients with $\mathrm{HE}$ [8], is associated with alterations in serotonin (5-HT)

*Corresponding author (email: jinfeng@psych.ac.cn) metabolic activity [9-11] resulting from HA [12]. Moreover, the kynurenine pathway (KP), which converts tryptophan (TRP) to L-kynurenine (KYN), may also be involved in modulating HA-induced anxiety disorder because TRP is the precursor for 5-HT synthesis [13-15].

Animal models of HA are crucial for elucidating potential pathophysiological mechanisms and thus, developing possible therapies for HE. Current animal models of chronic HA are generally limited to rats and mice, and have been designed to predominantly study the effects of HA per se on brain function [16]. The injection of ammonium acetate intraperitoneally (i.p.) has been shown to be successful in demonstrating HA-induced alterations in the brain [17-19]. These experiments are inexpensive and simple to perform. 
Classic therapeutic approaches for HE involve the reduction of HA levels via antibiotic treatment and administration of nonabsorbable sugars, such as lactulose [20,21]. These treatments, however, are not optimal therapies because of their side effects, toxicities, and poor compliance from patients [22]. Probiotics, such as the Lactobacillus species, are living microorganisms that contribute to the balance of intestinal microbiota, thereby improving the health of the host. Probiotics may have the potential to treat the neurological disorders associated with $\mathrm{HE}$, because of their ability to suppress the pro-inflammatory response $[23,24]$ and regulate the activity of the 5-HT system [25]. Indeed, data from animal and clinical studies have revealed that probiotics can improve cognitive function [26] and decrease anxiety and depression symptoms [25,27-29]. However, the mechanism by which probiotic bacteria residing in the gut enable their protective effects to the central nervous system remains unclear.

Therefore, the overall aim of this study was to assess the efficacy of the probiotic Lactobacillus helveticus (L. helveticus) strain NS8 in preventing cognitive impairment and anxiety-like behavior in HA rats. L. helveticus has been shown to inhibit pro-inflammatory responses and improve murine brain function and behavior [30,31].

\section{Materials and methods}

\subsection{Animals}

Specific-pathogen-free male Sprague-Dawley rats (180200 g) (Vital River Animal Centre, China) were used in the study. Rats were housed individually in wire-mesh cages in an animal room at a controlled temperature $\left(20 \pm 2^{\circ} \mathrm{C}\right)$, with a relative humidity of $50 \%-55 \%$, and exposed to a $12: 12 \mathrm{~h}$ light/dark cycle. Animals had ad libitum access to standard laboratory rodent chow and fresh sterile water. Three groups of animals (six rats per group) were used in the study: (i) sterile saline-injected (i.p.) rats exposed to fresh sterile water (control group), (ii) HA rats exposed to fresh sterile water (HA group), and (iii) HA rats exposed to fresh sterile water containing L. helveticus NS8 (HA+NS8 group). The experimental protocol was approved by the Animal
Experiment Ethics Committee of the Institute of Psychology, Chinese Academy of Sciences.

\subsection{HA rat model}

HA was induced by $2.5 \mathrm{mmol} \mathrm{kg}{ }^{-1}$ body weight ammonium acetate (Sigma-Aldrich, USA) (i.p.) dissolved in the sterile saline, once per day, three times per week for four consecutive weeks $[19,32]$.

\subsection{L. helveticus NS8 strain and culture conditions}

The L. helveticus strain NS8 (GenBank accession No. JQ013296.1) was isolated by our laboratory from natural fermented dairy products collected from grassland in Inner Mongolia, China. L. helveticus strain NS8 was stored in DeMan-Rogosa-Sharpe (MRS) broth (Biokar Diagnostics, France) at $-80^{\circ} \mathrm{C}$, and subcultured twice in MRS broth for $18 \mathrm{~h}$ at $37^{\circ} \mathrm{C}$ prior to its use in experiment. Overnight cultures were centrifuged twice at $1500 \times g$ for $5 \mathrm{~min}$, cells collected and resuspended in fresh sterile water $\left(10^{9} \mathrm{CFU} \mathrm{mL} \mathrm{m}^{-1}\right)$ [33]. NS8-containing drinking water (probiotic treatment) was given to HA rats over two weeks. A high bacterial viability was maintained by supplying fresh L. helveticus NS8-containing drinking daily. The daily dosages of probiotics ingested by the rats were measured by the daily amount of water consumed.

\subsection{Experimental design}

Two weeks acclimatization of all rats (week 1-2), ammonium acetate or saline was administered for four weeks (week 3-6) to induce chronic HA or serve as control, respectively (Figure 1). HA rats were then given L. helveticus NS8-containing drinking water or sterile water (week 7-8). Anxiety-like behavior and cognitive function were tested (at week 9) using the elevated plus maze (EPM) and Morris water maze (MWM), respectively. Animals continued to receive either ammonium acetate or sterile saline during probiotic administration and behavioral tests (week 7-9) to prevent spontaneous recovery from HA. During the behavioral tests, animals continued to drink either sterile water

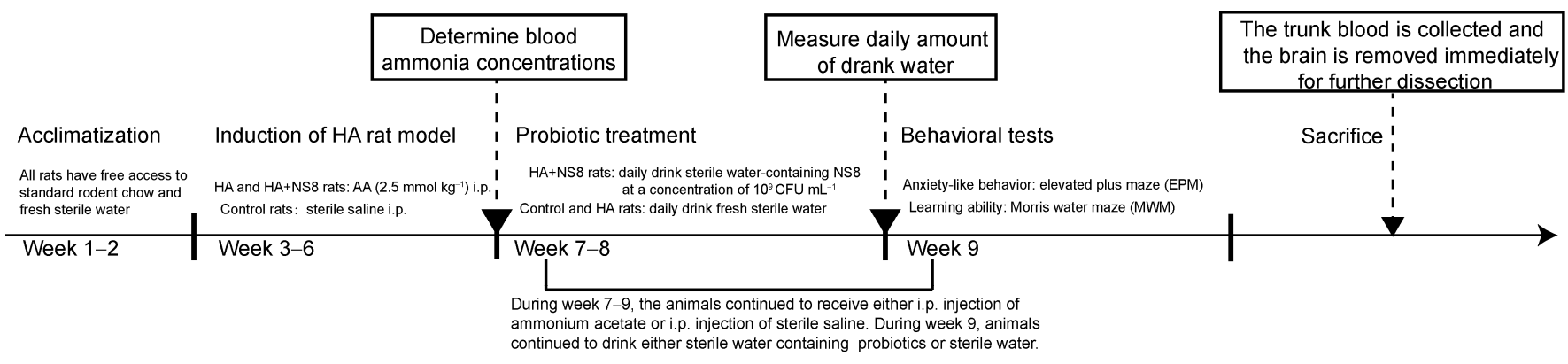

Figure 1 Experimental protocol design. Time-line of rat experimental procedure. HA, hyperammonemia; NS8, L. helveticus strain NS8; AA, ammonium acetate; i.p., intraperitoneal. 
containing L. helveticus NS8 or sterile water.

Blood samples $(20 \mu \mathrm{L})$ were collected (via a small tail incision) for the measurement of ammonia concentrations at the end of HA or saline treatment (week 6). The daily amount of water consumed was measured at the end of the two-week probiotic or water treatment (week 8). Rats were sacrificed by decapitation after the final MWM test. The trunk blood was collected and the brain was removed immediately for further dissection.

\subsection{The EPM test}

The EPM test is widely used to measure anxiety-like behavior in response to a novel environment and height. The time spent in and number of entries into the open arms were taken as indices of anxiety. These parameters were expressed as a percentage of the total time spent and the total entries into any arm during the 5 min test session [34].

The EPM consisted of a center area $(10 \mathrm{~cm} \times 10 \mathrm{~cm})$ with two opposite closed arms (a $10 \mathrm{~cm}$ wide, $50 \mathrm{~cm}$ long, and $30 \mathrm{~cm}$ high wall at their sides and far end) and two opposite open arms (10 cm wide, $50 \mathrm{~cm}$ long) arranged as a plus [34]. The device was made of opaque black polypropylene and elevated $50 \mathrm{~cm}$ above the floor.

Rats were placed individually in the center of the maze facing an open arm, and were allowed $5 \mathrm{~min}$ of free exploration. Movements of the animals during the 5-min test period were tracked by a video camera positioned above the center of the maze and were analyzed using the ANY-Maze (Stoelting, USA) video tracking system.

\subsection{The MWM task}

The Morris Water Maze consisted of a dark circular pool (140 $\mathrm{cm}$ in diameter and $55 \mathrm{~cm}$ high) filled (25 cm deep) with water $\left(20 \pm 1^{\circ} \mathrm{C}\right)$ [35]. The pool was divided into four zones (arbitrarily designated into north-east, south-east, south-west, and north-west orthogonal quadrants) by the ANY-Maze software (Stoelting, USA). Extra maze cues surrounded the walls of the room where the water maze was situated.

The MWM task lasted for $6 \mathrm{~d}$. During the first four days, rats were trained over four trials per day. The platform (11 $\mathrm{cm}$ in diameter) was submerged $1.5 \mathrm{~cm}$ from the water surface (and rendered invisible) in the center of the south-west quadrant. In each trial, rats were placed in front of the wall but at a different starting position (north, east, south and west). Rats were free to swim and find the hidden platform within $120 \mathrm{~s}$. The escape latency to find the hidden platform is taken as an index of learning ability [35]. On day 5, a spatial probe test was used to assess retention of spatial memory by the swim time in the target zone. For this test, the hidden platform was removed and rats were free to swim for $120 \mathrm{~s}$ from the north-east orthogonal quadrant of the pool, a starting position furthest away from the hidden platform. On day 6, a visible platform (not submerged in water) covered by a piece of aluminum foil was placed in another position (the south-east quadrant) to test swimming ability and motivation of the rats.

\subsection{Measurement of blood ammonia}

Blood ammonia levels were measured using a Blood Ammonia Checker AA-4120 (ARKRAY, Inc., Japan). The measuring range was $10-400 \mu \mathrm{g} \mathrm{dL}^{-1}$.

\subsection{Measurement of neuroinflammatory markers}

Measurements were carried out in the cerebellum, hippocampus and prefrontal cortex, which are brain regions known to be crucially involved in cognitive function and emotional state [36,37]. These brain regions were removed according to Desbonnet et al. [25]. Briefly, brain regions were quickly isolated on ice, weighed, then snapped frozen by liquid nitrogen and stored at $-80^{\circ} \mathrm{C}$ until analysis. Brain tissues were homogenized in phosphate buffered saline $(0.1$ mol L ${ }^{-1}$ ) containing a protease inhibitor cocktail (AMRESCOLLC, USA). The homogenate was centrifuged $(3000 \times g$, $10 \mathrm{~min}$, at $4^{\circ} \mathrm{C}$ ). Prostaglandin E2 (PGE2), inducible nitric oxide synthase (iNOS) and interleukin-1 beta (IL-1 $\beta$ ) were measured from the supernatant by enzyme-linked immunosorbent assay (ELISA) kits (RapidBio Lab, USA), according to the manufacturer's protocol.

\subsection{Measurement of the metabolic activity of brain 5-HT}

5-HT and its metabolite 5-hyroxyindole acetic acid (5-HIAA) were measured in the supernatant of the prefrontal cortex, hippocampus and cerebellum using ELISA kits (RapidBio Lab), according to the manufacturer's protocol.

\subsection{Analysis of the kynurenine pathway in plasma}

Trunk blood was collected in pre-chilled ethylenediaminetetraacetic acid-coated blood collection tubes, and plasma was separated by centrifugation $(1500 \times \mathrm{g}, 10 \mathrm{~min}$, at $4^{\circ} \mathrm{C}$ ) and immediately stored at $-80^{\circ} \mathrm{C}$ until analysis. Plasma levels of tryptophan (TRP), and its metabolites, L-kynurenine (KYN) and kynurenic acid (KA), were measured by ELISA kits (RapidBio Lab), according to the manufacturer's instructions.

\subsection{Statistical analysis}

All data are presented as the mean \pm SEM. The two-way analysis of variance (ANOVA) was used to identify training days 1-4 of MWM task, treatment effects, and interactions between these factors, and the other data were analyzed by one-way ANOVA. The homogeneity of variance was ana- 
lyzed with the Levene test. For post hoc analysis of group differences, the Tukey HSD test was employed. Probability values of $P<0.05$ were regarded as significant difference. Statistical evaluation of the results was performed by SPSS 17.0 (SPSS Inc., USA).

\section{Results}

\subsection{Daily amount of consumed water remained un- changed between all three groups}

The daily amount of ingested water measured two weeks after probiotic administration was similar between the three groups; control group: $41.3 \pm 1.7 \mathrm{~mL}$, HA group: $42.3 \pm 3.4 \mathrm{~mL}$, and HA+NS8 group: $48.3 \pm 4.1 \mathrm{~mL}$.

\subsection{HA treatment elevated blood ammonium levels}

Ammonium acetate treatment for four weeks significantly $(P<0.01)$ increased ammonium levels to $130.8 \pm 8.9 \mu \mathrm{g} \mathrm{dL}^{-1}$ and $147.8 \pm 12.5 \mu \mathrm{g} \mathrm{dL}^{-1}$ in the HA group and HA+NS8, respectively, compared with the control $\left(28.2 \pm 3.4 \mu \mathrm{g} \mathrm{dL}^{-1}\right)$.

\subsection{Probiotic treatment increased the percentage of the number of entries into the open arms of the EPM test in HA rats}

Both the percentages of time spent $(5.3 \% \pm 2.1 \%$ vs. $30.2 \% \pm 4.5 \%, P<0.05)$ in and the number of entries $(12.3 \% \pm 4.5 \%$ vs. $43.4 \% \pm 5.4 \%, P<0.05)$ into the open arms were significantly reduced in HA rats, compared with the control (Figure 2A and B). Probiotic treatment of HA rats significantly $(P<0.05)$ increased the percentage of number of entries into the open arms $(39.7 \% \pm 2.1 \%)$ (Figure 2B), but had no effect on the time spent in the open arms, compared with HA rats.

\subsection{Probiotic treatment improved learning and memory abilities of the MWM task in HA rats}

The escape latency to find the hidden platform was significant different $(P<0.01)$ among the three groups over training days 1-4 (Figure 3 ). Although the three groups were not significantly different on day 1 , the escape latency to find the hidden platform was significantly increased in HA rats compared with the control on day 2, day 3 and day 4 (day 2: $110.1 \pm 5.9$ s vs. $53.2 \pm 5.7 \mathrm{~s}, P<0.01$; day $3: 96.5 \pm 10.2$ s vs. $14.2 \pm 2.3 \mathrm{~s}, P<0.01$; day $4: 109.1 \pm 7.8$ s vs. $10.3 \pm 1.3 \mathrm{~s}$, $P<0.01)$. However, the escape latency was significantly $(P<0.01 ; P<0.01 ; P<0.01)$ reduced in HA rats with probiotic treatment during the training days (day $2: 45.9 \pm 4.4 \mathrm{~s}$, day 3 : $11.3 \pm 1.5 \mathrm{~s}$, day $4: 5.7 \pm 0.4 \mathrm{~s}$ ) compared to HA rats, suggesting that probiotic treatment enhances learning ability (Figure 3). The interaction between the factors training days and treatment effects was significant $(P<0.01)$.

The percentage of swim time in the target zone is taken as an index of memory retention. The percentage of swim time in the target zone was significantly $(P<0.01)$ reduced in HA rats $(22.7 \% \pm 0.7 \%)$ compared with control rats $(32.4 \% \pm 0.8 \%)$ (Table 1). However, probiotic treatment sig-
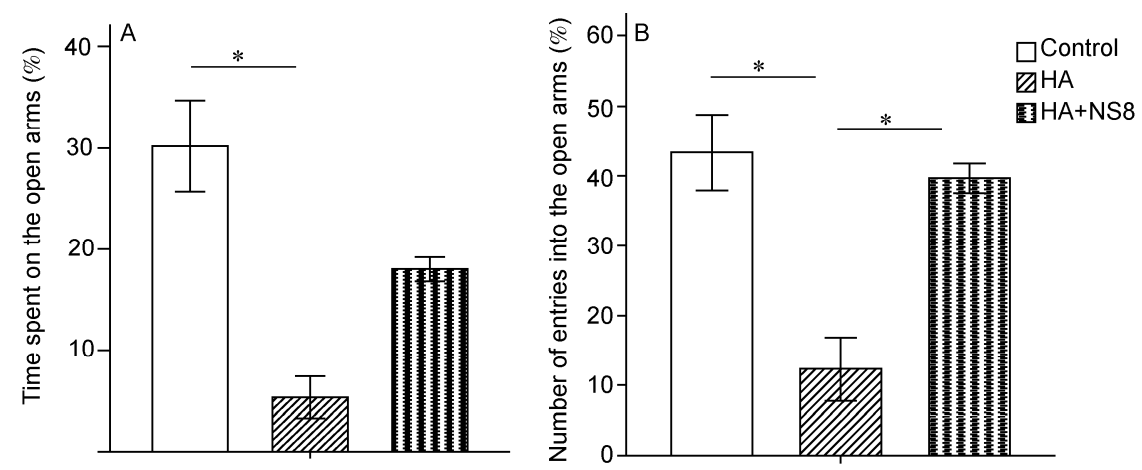

Figure 2 Probiotic treatment in HA rats prevents HA-induced anxiety-like behavior in the elevated plus maze (EPM) test. The time spent in and number of entries into the open arms were taken as indices of anxiety. A, Percentage of time spent in the open arms of the EPM. B, Percentage of the number of entries into the open arms of the EPM. Values are represented as the mean \pm SEM ( $n=6$ rats per group). *, $P<0.05$.

Table 1 Memory retention and swimming ability in the Morris water maze task ${ }^{\text {a) }}$

\begin{tabular}{cccc}
\hline & $\begin{array}{c}\text { Percentage of swimming time spent in the } \\
\text { target zone }(\mathrm{s})\end{array}$ & $\begin{array}{c}\text { Escape latency to the visible platform (s) } \\
\text { Swimming speed (cm s }\end{array}$ \\
\hline Control & $32.4 \pm 0.8$ & $5.2 \pm 0.2$ & $10.0 \pm 0.4$ \\
HA & $22.7 \pm 0.7^{* *}$ & $5.2 \pm 0.6$ & $10.1 \pm 0.8$ \\
HA+NS8 & $35.5 \pm 1.2^{\# \#}$ & $4.9 \pm 0.3$ & $10.4 \pm 0.8$ \\
ANOVA & $P<0.01$ & $P>0.05$ & $P>0.05$ \\
\hline
\end{tabular}

a) Memory retention is measured by the percentage of swim time in the target zone on training day 5 . Swimming ability is evaluated by the escape latency to find the visible platform and the swim speed on day 6 . Values are represented as the mean \pm SEM ( $n=6$ rats per group). HA, hyperammonemia; NS8, $L$. helveticus strain NS8; ANOVA, analysis of variance. **, $P<0.01$ vs. control; \#\#, $P<0.01$ vs. HA. 


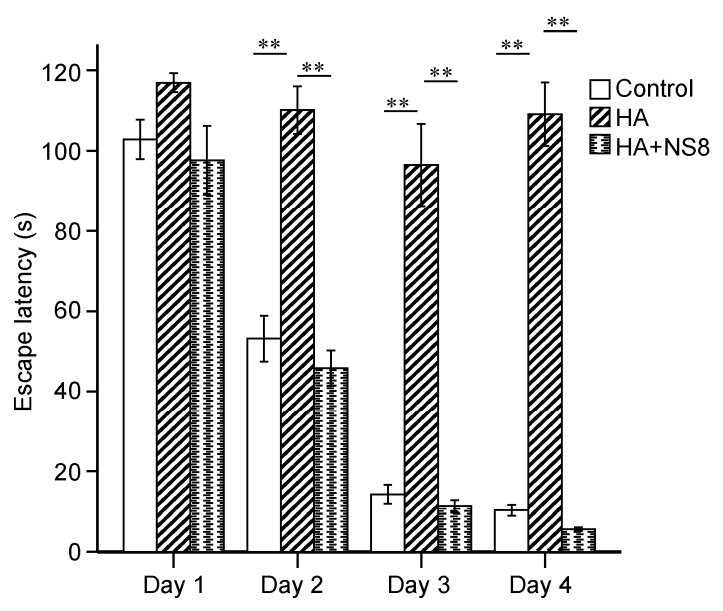

Figure 3 Probiotic treatment in HA rats restores HA-mediated impairment of the learning ability in Morris water maze (MWM) task. The escape latency to find the hidden platform over training days 1-4 was taken as an index of learning ability. Values are represented as the mean \pm SEM $(n=6$ rats per group). ${ }^{* *}, P<0.01$.

nificantly $(P<0.01)$ increased the percentage of swim time to $35.5 \% \pm 1.2 \%$, which is significantly greater than in HA rats (Table 1). The escape latency to find the visible platform and swim speed were not significantly different be- tween the three groups (Table 1); thus, swimming ability was unaffected.

\subsection{Probiotic treatment reduced the levels of PGE2

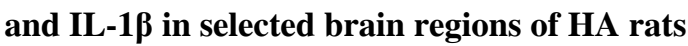

HA treatment significantly increased the level of iNOS in the cerebellum $\left(25.5 \pm 1.5 \mathrm{U} \mathrm{g}^{-1}\right.$ vs. $\left.19.5 \pm 1.0 \mathrm{U} \mathrm{g}^{-1}, P<0.01\right)$, but not in the hippocampus or prefrontal cortex, compared with control rats (Figure 4A). Probiotic treatment of HA rats had no significant effect on iNOS in any of these brain regions, compared with HA rats.

HA treatment significantly increased the concentration of PGE2 in the cerebellum $\left(2204.0 \pm 36.5 \mathrm{pg} \mathrm{g}^{-1}\right.$ vs. $1787.0 \pm$ $\left.59.5 \mathrm{pg} \mathrm{g}^{-1}, P<0.01\right)$ and hippocampus $\left(2282.0 \pm 83.0 \mathrm{pg} \mathrm{g}^{-1}\right.$ vs. $\left.1901.0 \pm 95.0 \mathrm{pg} \mathrm{g}^{-1}, P<0.05\right)$, but not in the prefrontal cortex, compared with the control (Figure 4B). Probiotic treatment of HA rats significantly reduced the concentrations of PGE2 in the cerebellum $(P<0.01)$ and hippocampus $(P<0.01)$ to $1560.5 \pm 162.5 \mathrm{pg} \mathrm{g}^{-1}$ and $1688.0 \pm 44.5 \mathrm{pg} \mathrm{g}^{-1}$, respectively, compared with HA rats (Figure 4B).

HA treatment significantly increased the concentration of IL-1 $\beta$ in the cerebellum $\left(175 \pm 8.0 \mathrm{pg} \mathrm{g}^{-1}\right.$ vs. $119.0 \pm 4.5 \mathrm{pg}$ $\left.\mathrm{g}^{-1}, P<0.01\right)$, hippocampus $\left(183.5 \pm 10.0 \mathrm{pg} \mathrm{g}^{-1}\right.$ vs. $134.0 \pm$
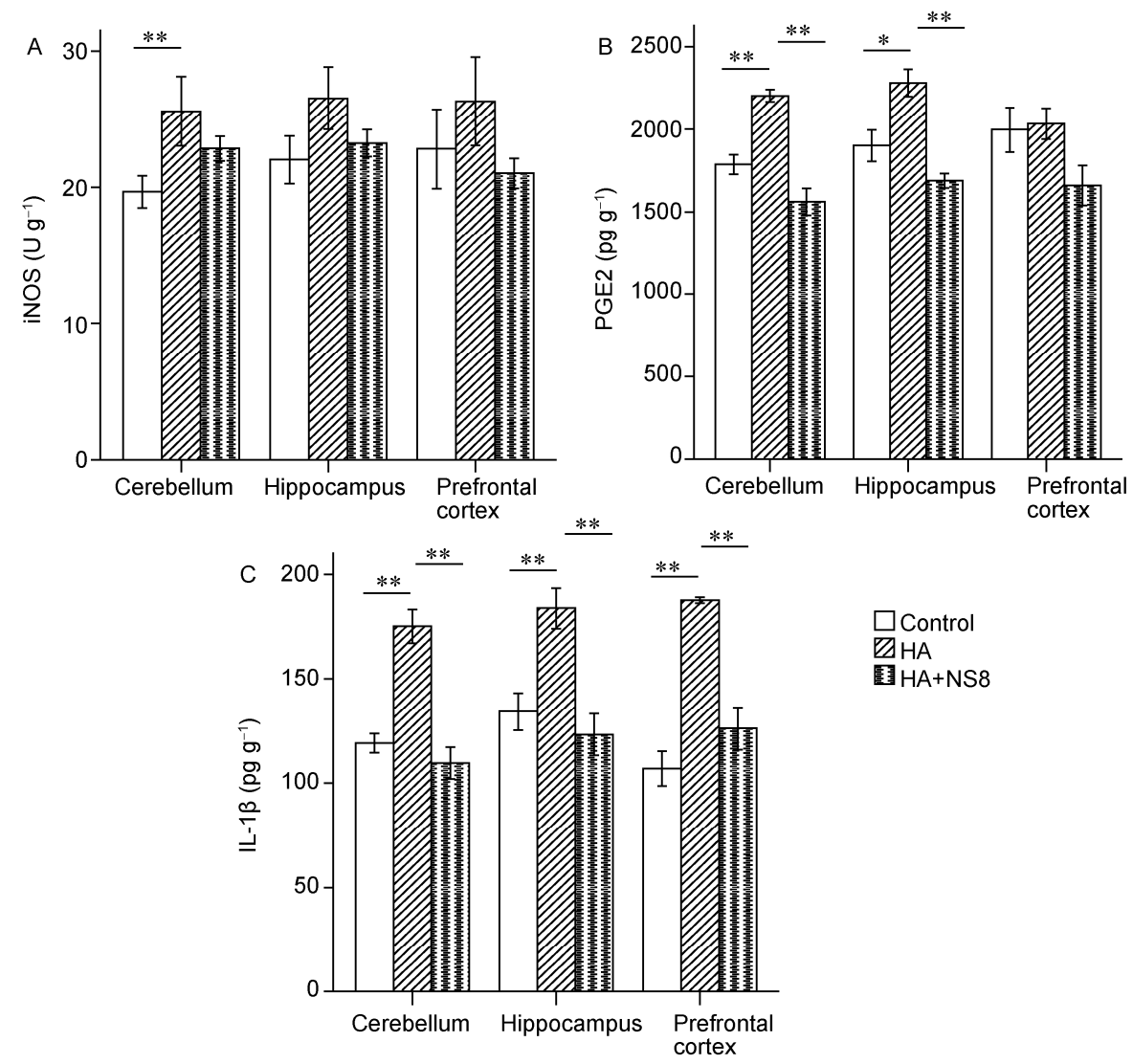

Figure 4 Probiotic treatment in HA rats attenuates HA-mediated neuroinflammation. Neuroinflammation was assessed by measuring the inflammatory markers: inducible nitric oxide synthase (iNOS) (A), prostaglandin E2 (PGE2) (B), and interleukin-1 beta (IL-1 $\beta$ ) (C) in the cerebellum, hippocampus and prefrontal cortex. Values are represented as the mean $\pm \operatorname{SEM}(n=6$ rats per group). *, $P<0.05 ; * *, P<0.01$. 
$\left.9.0 \mathrm{pg} \mathrm{g}^{-1}, P<0.01\right)$ and prefrontal cortex $\left(187.5 \pm 1.5 \mathrm{pg} \mathrm{g}^{-1}\right.$ vs. $\left.107.0 \pm 8.5 \mathrm{pg} \mathrm{g}^{-1}, P<0.01\right)$, compared to control rats (Figure 4C). Probiotic treatment of HA rats significantly reduced this level to $109.5 \pm 7.5,123.0 \pm 10.0$, and $126.0 \pm 10.0$ $\mathrm{pg} \mathrm{g}^{-1}$ in the cerebellum $(P<0.01)$, hippocampus $(P<0.01)$, and prefrontal cortex $(P<0.01)$, respectively, compared to HA rats (Figure $4 \mathrm{C}$ ).

\subsection{Probiotic treatment reduced levels of 5-HT but not its metabolite, 5-HIAA, in HA rats}

HA did not affect the level of 5-HT in any of the three tested brain regions (Figure 5A). However, HA significantly increased the concentrations of 5-HIAA in the cerebellum (77.5 $\pm 4.0 \mathrm{pg} \mathrm{g}^{-1}$ vs. $\left.50.0 \pm 4.0 \mathrm{pg} \mathrm{g}^{-1}, P<0.01\right)$, hippocampus (78.5 $\pm 5.0 \mathrm{pg} \mathrm{g}^{-1}$ vs. $\left.56.0 \pm 5.5 \mathrm{pg} \mathrm{g}^{-1}, P<0.05\right)$ and prefrontal cortex $\left(75.0 \pm 4.0 \mathrm{pg} \mathrm{g}^{-1}\right.$ vs. $\left.56.0 \pm 4.5 \mathrm{pg} \mathrm{g}^{-1}, P<0.05\right)$, compared with the control (Figure 5B). Probiotic treatment of HA rats did not affect 5-HIAA levels in any of the tested brain regions (Figure $5 \mathrm{~B})$, but significantly $(P<0.01)$ reduced the concentration of 5-HT in the cerebellum and hippocampus, compared with HA rats (Figure 5A).

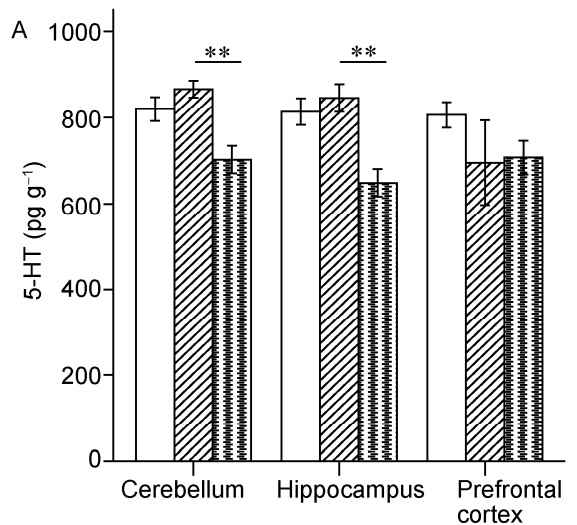

\subsection{Probiotic treatment of $\mathrm{HA}$ rats affected the plasma kynurenine pathway}

HA treatment significantly increased plasma levels of TRP $\left(30280 \pm 1089\right.$ pmol $\mathrm{mL}^{-1}$ vs. $23590 \pm 1260 \mathrm{pmol} \mathrm{mL}^{-1}$, $P<0.01$ ), compared with the control (Figure 6A). Furthermore, HA treatment significantly decreased KYN/TRP $(0.0121 \pm 0.00061$ vs. $0.0253 \pm 0.001, P<0.01)$, and increased $\mathrm{KA} / \mathrm{KYN}$ ratio $(1.4 \pm 0.09$ vs. $0.8 \pm 0.1, P<0.01)$, compared with the control (Figure 6B). Probiotic treatment of HA rats markedly increased $(P<0.01)$ and decreased $(P<0.05)$ the ratio of KYN/TRP $(0.022 \pm 0.001)$ and KA/KYN $(0.9 \pm 0.06)$, respectively, compared with HA rats (Figure 6B).

\section{Discussion}

In the present study, chronic HA induces cognitive decline and anxiety-like behavior in rats, supporting the idea that HA-mediate HE leads to neurological dysfunctions. Furthermore, probiotic treatment of HA rats with the L. helveticus NS8 improves cognitive decline and anxiety-like behavior, suggesting that this probiotic strain may be benefi-

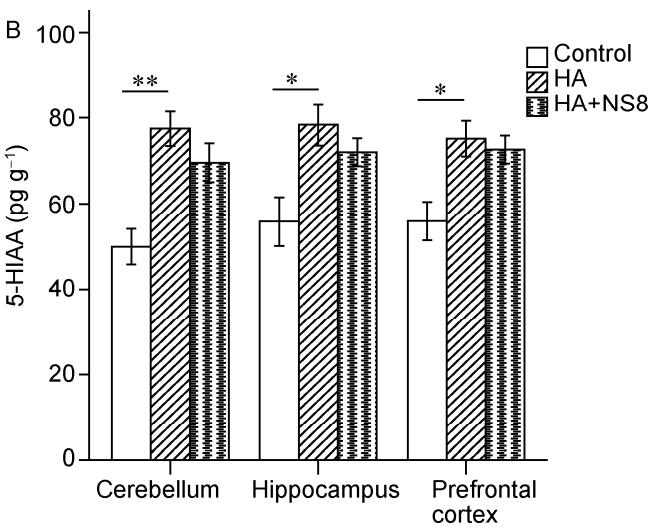

Figure 5 Probiotic treatment in HA rats restores the HA-mediated enhancement of 5-HT metabolic activity. The levels of 5-HT (A) and its metabolite, 5 -HIAA (B), in the cerebellum, hippocampus and prefrontal cortex. Values are represented as the mean \pm SEM ( $n=6$ rats per group). *, $P<0.05 ; * *, P<0.01$.
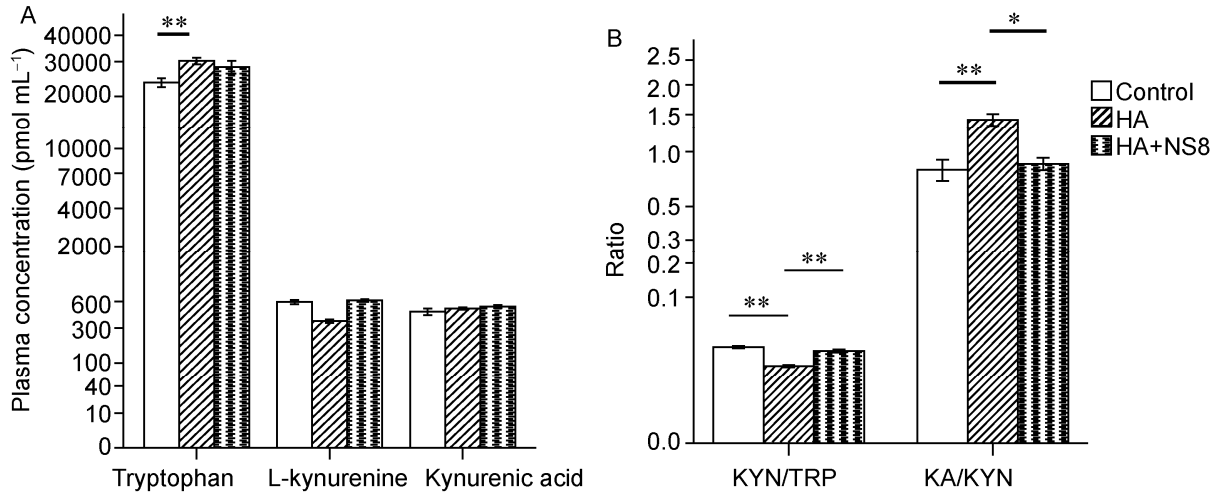

Figure 6 Probiotic treatment in HA rats restores HA-induced alterations in the kynurenine pathway. Plasma concentrations of tryptophan (TRP), L-kynurenine $(\mathrm{KYN})$ and kynurenic acid $(\mathrm{KA})(\mathrm{A})$, and the KYN/TRP and KA/KYN ratio (B). Values are represented as the mean \pm SEM $(n=6$ rats per group). *, $P<0.05$; **, $P<0.01$. 
cial for the treatment of neurological dysfunctions in HA rats.

\subsection{Effect of L. helveticus NS8 on cognitive function and neuroinflammation}

The mechanism by which HA impairs cognitive function is beginning to be clarified in animal studies. Portacaval shunts (PCS) rats have been found to develop learning impairment of the Y-maze task. These rats exhibit HA and neuroinflammation [36]. Treatment of PCS rats with the anti-inflammatory, ibuprofen, reduces neuroinflammation but not HA, and also restores learning ability [38]. Furthermore, data from a rat model of chronic HA have suggested that neuroinflammation mediates the deleterious effects of HA on cognitive function [1]. These results suggest that HA may induce neuroinflammation-mediated cognitive impairment. In support of this pathogenic role, our study shows that HA induces neuroinflammation in the cerebellum, hippocampus and prefrontal cortex, and impairs learning ability and spatial memory in rats. Therefore, probiotic treatment may be beneficial for HA-mediated cognitive decline in rats given because cognitive decline is improved and neuroinflammation is attenuated with this treatment.

A main mechanism of HA-induced neuroinflammation is the release of pro-inflammatory molecules from activated microglia [1]. Moreover, the transcription factor, nuclear factor kappa B $(\mathrm{NF}-\kappa \mathrm{B})$, plays a critical role in microglial activation [39]. Therefore, in the present study, probiotic treatment may have attenuated neuroinflammation by suppressing the activation of NF- $\kappa \mathrm{B}$. In support of this hypothesis, related studies have reported that suppression of inflammation can occur in response to probiotic Lactobacillus-mediated inhibition of NF- $\kappa \mathrm{B}$ activation [40,41].

\subsection{Effect of $L$. helveticus NS8 on anxiety-like behavior and 5-HT metabolism}

A dysfunctional of 5-HT system has long been implicated in the pathogenesis of anxiety disorders [42]. Thus, the anxiety-like behavior observed in HA rats of the present study may have resulted from changes in the central 5-HT system. In support of this theory, our results showed that HA rats had enhanced 5-HT metabolism in the cerebellum, hippocampus and prefrontal cortex, as reflected by increased levels of 5-HIAA but unchanged levels of 5-HT. A previous study found similar results in PCS-induced HA rats that displayed elevated 5-HIAA but unaltered 5-HT levels, and when challenged with ammonium acetate, 5-HT release was transiently elevated $[9,12]$.

Indeed, results from related studies have suggested an association between the hyperactivity of the 5-HT system and an increased susceptibility to develop an anxiety disorder. Iversen [43] proposed that an increase in brain 5-HT concentration elevates anxiety while a decrease in brain
5-HT level reduces anxiety. Moreover, reduced wholetissue level of 5-HT in regional brain was linked to a low-anxiety phenotype in transgenic mice overexpressing 5-HT transporter [44]. Therefore, the results observed in the present study, that L. helveticus NS8 reduces the levels of 5-HT in the cerebellum and hippocampus, and improves anxiety-like behavior in HA rats, may suggest that this probiotic may be beneficial in the treatment of anxiety disorder in HA rats.

\subsection{Effect of L. helveticus NS8 on kynurenine pathway}

5-HT synthesis in the brain is crucially dependent on its precursor TRP. Therefore, enhanced 5-HT metabolism in HA rats of the present study may be attributed to a significant rise in TRP. Peripheral TRP concentration is increased and correlates well with central TRP, 5-HT and 5-HIAA levels in HE patients $[10,45]$. L. helveticus NS8-mediated reduction of 5-HT metabolism in HA rats in the current study was possibly due to enhanced TRP degradation because the KYN/TRP ratio was increased, despite the levels of TRP themselves remaining unchanged.

The kynurenine pathway is the major route for TRP metabolism, in which indoleamine 2,3-dioxygenase (IDO) converts TRP to KYN [46]. In the current study, the rise in the KYN/TRP ratio in HA rats treated with probiotics may indicate that probiotics enhance IDO activity. IDO is expressed in various cell types, and is widely involved in immunomodulatory activity [47], such as anti-inflammatory effect and protective role in a mouse model of colitis [48]. Therefore, in the present study, improved anxiety-like behavior in probiotic treatment of HA rats may be a result of an immunomodulatory effect of this treatment through enhanced IDO activity, the promotion of TRP depletion, and reduction of 5-HT metabolism. Similarly in another study, attenuation of the allergic airway response was found to be due to an increase in systemic IDO activity induced by administration of live Lactobacillus reuteri [49]. However, our results are contradicted by other studies that have shown suppression of IDO production in response to treatment of the probiotic Bifidobacterium species or Lactobacillus johnsonii in a rat model of depression [25,50]. In the mouse model of allergic airway inflammation, it is important to note that the IDO activity was enhanced only following the administration of Lactobacillus reuteri but not Lactobacillus salivarius [49]. These data suggest that different probiotic species or strains may have different effects on IDO activity. Furthermore, KA produced from KYN has been shown to enhance anxiety because it can decrease the extracellular level of glutamate [51,52]. Results from our study show an increase in the $\mathrm{KA} / \mathrm{KYN}$ ratio in HA rats but a decrease of that following probiotic treatment, thus providing further evidence for the potential anxiolytic properties of the probiotic L. helveticus strain NS8.

In conclusion, the data from our study suggest that ad- 
ministration of probiotic L. helveticus strain NS8 is a potential therapeutic approach for HA-mediated cognitive decline and anxiety-like behavior. The effect of probiotic treatment on improving behaviors may be attributed to its immunomodulatory properties of attenuating neuroinflammation and reducing 5-HT metabolism. We therefore provide strong evidence supporting the possible use of probiotic treatment for neurological disorders in addition to its use in the gastrointestinal tract. A better understanding of the behavioral effect of probiotics would require further investigations of their immunomodulatory activity.

\section{The authors declare that no competing financial interests exist.}

This work was supported by NS Bio Japan and NS Health Biotechnology Beijing.

1 Rodrigo R, Cauli O, Gomez-Pinedo U, Agusti A, Hernandez-Rabaza V, Garcia-Verdugo JM, Felipo V. Hyperammonemia induces neuroinflammation that contributes to cognitive impairment in rats with hepatic encephalopathy. Gastroenterology, 2010, 139: 675-684

2 Saul WSWB. Hyperammonemic encephalopathy. Medicine (Abingdon), 2002, 81: 240-249

3 Felipo V, Butterworth RF. Neurobiology of ammonia. Prog Neurobiol, 2002, 67: 259-279

4 Lajtha A, Reith MEA, eds. Handbook of Neurochemistry and Molecular Neurobiology. 3rd ed. New York: Springer, 2008

5 Basile AS, Jones EA. Ammonia and GABA-ergic neurotransmission: Interrelated factors in the pathogenesis of hepatic encephalopathy. Hepatology, 1997, 25: 1303-1305

6 Montoliu C, Piedrafita B, Serra MA, del Olmo JA, Urios A, Rodrigo JM, Felipo V. IL-6 and IL-18 in blood may discriminate cirrhotic patients with and without minimal hepatic encephalopathy. J Clin Gastroenterol, 2009, 43: 272-279

7 Gibertini M, Newton C, Friedman H, Klein TW. Spatial learning impairment in mice infected with legionella pneumophila or administered exogenous interleukin-1 $\beta$. Brain Behav Immun, 1995, 9: $113-128$

8 Wiltfang J, Nolte W, Weißenborn K, Komhuber J, Rüther E. Psychiatric aspects of portal-systemic encephalopathy. Metab Brain Dis, 1998, 13: 379-389

9 Erecinska M, Pastuszko A, Wilson DF, Nelson D. Ammonia-induced release of neurotransmitters from rat brain synaptosomes: differences between the effects on amines and amino acids. J Neurochem, 1987, 49: 1258-1265

10 Rössle M, Luft M, Herz R, Klein B, Lehmann M, Gerok W. Amino acid, ammonia and neurotransmitter concentrations in hepatic encephalopathy: serial analysis in plasma and cerebrospinal fluid during treatment with an adapted amino acid solution. Klin Wochenschr, 1984, 62: 867-875

11 Murphy DL, Moya PR, Fox MA, Rubenstein LM, Wendland JR, Timpano KR. Anxiety and affective disorder comorbidity related to serotonin and other neurotransmitter systems: obsessive-compulsive disorder as an example of overlapping clinical and genetic heterogeneity. Philos Trans R Soc Lond B Biol Sci, 2013, 368: 20120435

12 Bergqvist PBF, Hjorth S, Audet RM, Apelqvist G, Bengtsson F, Butterworth RF. Ammonium acetate challenge in experimental chronic hepatic encephalopathy induces a transient increase of brain 5-HT release in vivo. Eur Neuropsychopharm, 1996, 6: 317-322

13 Miura H, Ozaki N, Sawada M, Isobe K, Ohta T, Nagatsu T. A link between stress and depression: shifts in the balance between the kynurenine and serotonin pathways of tryptophan metabolism and the etiology and pathophysiology of depression. Stress, 2008, 11:
198-209

14 Vignau J, Costisella O, Canva V, Imbenotte M, Duhamel A, Lhermitte M. Impact of interferon alpha immunotherapy on tryptophan metabolism in patients with chronic hepatitis C. Results of a pilot studies on ten patients. Encephale, 2009, 35: 477-483

15 Laugeray A, Launay JM, Callebert J, Surget A, Belzung C, Barone PR. Peripheral and cerebral metabolic abnormalities of the tryptophan-kynurenine pathway in a murine model of major depression. Behav Brain Res, 2010, 210: 84-91

16 Butterworth RF, Norenberg MD, Felipo V, Ferenci P, Albrecht J, Blei AT, Members of the ICoEMoHE. Experimental models of hepatic encephalopathy: ISHEN guidelines. Liver Int, 2009, 29: 783-788

17 Lena PJ, Subramanian P. Effects of melatonin on the levels of antioxidants and lipid peroxidation products in rats treated with ammonium acetate. Pharmazie, 2004, 59: 636-639

18 Moroni F, Lombardi G, Moneti G, Cortesini C. The release and neosynthesis of glutamic acid are increased in experimental models of hepatic encephalopathy. J Neurochem, 1983, 40: 850-854

19 Subash S, Subramanian P. Morin a flavonoid exerts antioxidant potential in chronic hyperammonemic rats: a biochemical and histopathological study. Mol Cell Biochem, 2009, 327: 153-161

20 Bajaj JS, Sanyal AJ, Bell D, Gilles H, Heuman DM. Predictors of the recurrence of hepatic encephalopathy in lactulose-treated patients. Aliment Pharm Therap, 2010, 31: 1012-1017

21 Flamm SL. Rifaximin treatment for reduction of risk of overt hepatic encephalopathy recurrence. Therap Adv Gastroenterol, 2011, 4: 199-206

22 Nicaise C, Prozzi D, Viaene E, Moreno C, Gustot T, Quertinmont E, Demetter P, Suain V, Goffin P, Devière J, Hols P. Control of acute, chronic, and constitutive hyperammonemia by wild-type and genetically engineered Lactobacillus plantarum in rodents. Hepatology, 2008, 48: 1184-1192

23 Isolauri E, Sütas Y, Kankaanp P, Arvilommi H, Salminen S. Probiotics: effects on immunity. Am J Clin Nutr, 2001, 73: 444S-450S

24 Steidler L, Hans W, Schotte L, Neirynck S, Obermeier F, Falk W, Fiers W, Remaut E. Treatment of murine colitis by Lactococcus lactis secreting interleukin-10. Science, 2000, 289: 1352-1355

25 Desbonnet L, Garrett L, Clarke G, Bienenstock J, Dinan TG. The probiotic Bifidobacteria infantis: an assessment of potential antidepressant properties in the rat. J Psychiat Res, 2008, 43: 164-174

26 Gareau MG, Wine E, Rodrigues DM, Cho JH, Whary MT, Philpott DJ, MacQueen G, Sherman PM. Bacterial infection causes stress-induced memory dysfunction in mice. Gut, 2011, 60: 307-317

27 Benton D, Williams C, Brown A. Impact of consuming a milk drink containing a probiotic on mood and cognition. Eur J Clin Nutr, 2007, 61: 355-361

28 Rao AV, Bested AC, Beaulne TM, Katzman MA, Iorio C, Berardi JM, Logan AC. A randomized, double-blind, placebo-controlled pilot study of a probiotic in emotional symptoms of chronic fatigue syndrome. Gut Pathog, 2009, 1: 6

29 Bravo JA, Forsythe P, Chew MV, Escaravage E, Savignac HM, Dinan TG, Bienenstock J, Cryan JF. Ingestion of Lactobacillus strain regulates emotional behavior and central GABA receptor expression in a mouse via the vagus nerve. Proc Natl Acad Sci USA, 2011, 108: 16050-16055

30 Joo HM, Kim KA, Myoung KS, Ahn YT, Lee JH, Huh CS, Han MJ, Kim DH. Lactobacillus helveticus HY7801 ameliorates vulvovaginal candidiasis in mice by inhibiting fungal growth and NF- $\kappa$ B activation. Int Immunopharmacol, 2012, 14: 39-46

31 Ohland CL, Kish L, Bell H, Thiesen A, Hotte N, Pankiv E, Madsen KL. Effects of Lactobacillus helveticus on murine behavior are dependent on diet and genotype and correlate with alterations in the gut microbiome. Psychoneuroendocrinology, 2013, 38:1738-1747

32 Gokcimen A, Kocak A, Gulle K, Sutcu R, Elmas O, Caliskan S, Ozguner F. The effects of allopurinol on rat liver and spleen tissues in a chronic hyperammonemia animal model. Saudi Med J, 2007, 28: 
1648-1653

33 Sgouras D, Maragkoudakis P, Petraki K, Martinez-Gonzalez B, Eriotou E, Michopoulos S, Kalantzopoulos G, Tsakalidou E, Mentis A. In vitro and in vivo inhibition of helicobacter pylori by lactobacillus casei strain shirota. Appl Environ Microb, 2004, 70: 518-526

34 Walf AA, Frye CA. The use of the elevated plus maze as an assay of anxiety-related behavior in rodents. Nat Protocols, 2007, 2: 322-328

35 Wolfer DP, Stagljar-Bozicevic M, Errington ML, Lipp HP. Spatial memory and learning in transgenic mice: fact or artifact? Physiology, 1998, 13: 118-123

36 Monfort P, Cauli O, Montoliu C, Rodrigo R, Llansola M, Piedrafita B, el Mlili N, Boix J, Agustí A, Felipo V. Mechanisms of cognitive alterations in hyperammonemia and hepatic encephalopathy: therapeutical implications. Neurochem Int, 2009, 55: 106-112

37 Reznikov L, Fadel J, Reagan L. Glutamate-mediated neuroplasticity deficits in mood disorders. Neuroplasticity, 2009, 13-26

38 Cauli O, Rodrigo R, Piedrafita B, Boix J, Felipo V. Inflammation and hepatic encephalopathy: Ibuprofen restores learning ability in rats with portacaval shunts. Hepatology, 2007, 46: 514-519

39 Khasnavis S, Jana A, Roy A, Wood T, Ghosh S, Watson R, Pahan K. Suppression of nuclear factor- $\kappa \mathrm{B}$ activation and inflammation in microglia by a physically-modified saline. J Biol Chem, 2012, 287: 29529-29542

40 Riedel CU, Foata F, Philippe D, Adolfsson O, Eikmanns BJ, Blum S. Anti-inflammatory effects of bifidobacteria by inhibition of LPSinduced NF- $\mathrm{KB}$ activation. World J Gastroenterol, 2006, 12: 3729-3735

41 Enck P, Klosterhalfen S, Martens U. Probiotic therapy for irritable bowel syndrome. Dtsch Med Wochenschr, 2011, 136: 371-375

42 Laugeray A, Launay JM, Callebert J, Surget A, Belzung C, Barone PR. Evidence for a key role of the peripheral kynurenine pathway in the modulation of anxiety- and depression-like behaviours in mice: focus on individual differences. Pharmacol Biochem Be, 2011, 98: 161-168

43 Iversen SD. 5-HT and anxiety. Neuropharmacology 1984, 23: 15531560
44 Jennings KA, Loder MK, Sheward WJ, Pei Q, Deacon RMJ, Benson MA, Olverman HJ, Hastie ND, Harmar AJ, Shen S, Sharp T. Increased expression of the 5-HT transporter confers a low-anxiety phenotype linked to decreased 5-HT transmission. J Neurosci, 2006, 26: 8955-8964

45 Dejong CH, van de Poll MC, Soeters PB, Jalan R, Olde Damink SW. Aromatic amino acid metabolism during liver failure. J Nutr, 2007, 137: 1579S-1585S

46 O'Connor JC, André C, Wang Y, Lawson MA, Szegedi SS, Lestage J, Castanon N, Kelley KW, Dantzer R. Interferon- $\gamma$ and tumor necrosis factor- $\alpha$ mediate the upregulation of indoleamine 2,3-dioxygenase and the induction of depressive-like behavior in mice in response to bacillus calmette-guérin. J Neurosci, 2009, 29: 4200-4209

47 Däubener W, Schmidt SK, Heseler K, Spekker KH, MacKenzie CR. Antimicrobial and immunoregulatory effector mechanisms in human endothelial cells. Indoleamine 2,3-dioxygenase versus inducible nitric oxide synthase. Thromb Haemost, 2009, 102: 1110-1116

48 Gurtner GJ, Newberry RD, Schloemann SR, McDonald KG, Stenson WF. Inhibition of indoleamine 2,3-dioxygenase augments trinitrobenzene sulfonic acid colitis in mice. Gastroenterology, 2003, 125: 1762-1773

49 Forsythe P, Inman MD, Bienenstock J. Oral treatment with live Lactobacillus reuteri inhibits the allergic airway response in mice. Am J Respir Crit Care Med, 2007, 15: 561-569

50 Valladares R, Bojilova L, Potts AH, Cameron E, Gardner C, Lorca G, Gonzalez CF. Lactobacillus johnsonii inhibits indoleamine 2,3dioxygenase and alters tryptophan metabolite levels in BioBreeding rats. FASEB J, 2013, 27: 1711-1720

51 Wu HQ, Pereira ER, Bruno J, Pellicciari R, Albuquerque E, Schwarcz R. The astrocyte-derived $\alpha 7$ nicotinic receptor antagonist kynurenic acid controls extracellular glutamate levels in the prefrontal cortex. J Mol Neurosci, 2010, 40: 204-210

52 Cortese BM, Mitchell TR, Galloway MP, Prevost KE, Fang J, Moore GJ, Uhde TW. Region-specific alteration in brain glutamate: possible relationship to risk-taking behavior. Physiol Behav, 2010, 99: $445-450$

Open Access This article is distributed under the terms of the Creative Commons Attribution License which permits any use, distribution, and reproduction in any medium, provided the original author(s) and source are credited. 